\title{
Monte Carlo modelling of daylight activated photodynamic therapy
}

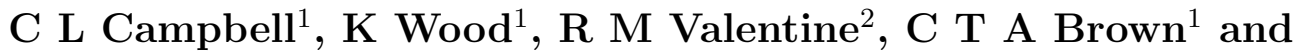 \\ H Moseley ${ }^{2}$
}

${ }^{1}$ School of Physics and Astronomy, University of St Andrews, UK

${ }^{2}$ Photobiology Unit, Ninewells Hospital and Medical School, University of Dundee, UK

E-mail: clc57@st-andrews.ac.uk

January 2014

\begin{abstract}
The treatment of superficial skin lesions via daylight activated photodynamic therapy (PDT) has been explored theoretically with three dimensional (3D) Monte Carlo radiation transfer (MCRT) simulations. For similar parameters and conditions, daylight activated PDT was compared to conventional PDT using a commercially available light source. Under reasonable assumptions for the optical properties of the tissue, protoporphyrin IX (PpIX) concentration, and a treatment dose of $75 \mathrm{~J} \mathrm{~cm}^{-2}$, it was found that during a clear summer day an effective treatment depth of over $2 \mathrm{~mm}$ can be achieved after 30 min of daylight illumination at a latitude of 56 degrees North. The same light dose would require $2.5 \mathrm{~h}$ of daylight illumination during an overcast summer day where a treatment depth of about $2 \mathrm{~mm}$ can be achieved. For conventional PDT the developed model suggests that $15 \mathrm{~min}$ of illumination is required to deliver a light dose of $75 \mathrm{Jcm}^{-2}$, which would result in an effective treatment depth of about $3 \mathrm{~mm}$. The model developed here allows for the determination of phototoxicity in skin tissue as a function of depth for different weather conditions as well as for conventional light sources. Our theoretical investigation supports clinical studies and shows that daylight activated PDT has the potential for treating superficial skin lesions during different weather conditions.
\end{abstract}

\section{Introduction and Background}

Photodynamic therapy (PDT) relies on the interaction between light, molecular oxygen and a photosensitive chemical to produce singlet oxygen and other reactive oxygen species (ROS), leading to tissue injury and death of targeted cells (Henderson and Dougherty, 1992; Castano et al., 2004). PDT is an efficient treatment method and is commonly used to treat non melanoma skin cancers (NMSC) and precancerous lesions such as aktinic keratosis (AKs) (Wilson and Patterson, 2008). However, PDT is often

associated with pain during light illumination (Castano et al., 2004). The prodrug 5-aminolaevulinic acid (ALA) or its methyl ester, methyl aminolevulinate (MAL) is 
administered topically in the form of a cream. The drug then diffuses through the skin and is converted (at a higher rate by the lesional cells) to the photosensitive chemical protoporhyrin IX (PpIX) via the heme biosynthetic pathway (Wachowska et al., 2011; Darlenski and Fluhr, 2012), resulting in a high accumulation and localisation of PpIX in the affected lesional areas.

PpIX absorbs light over a wide range of the visible spectrum and therefore a large range of different artificial light sources are suitable for treatment. DaylightPDT removes the need for artificial light sources, results in less pain during treatment (Cottrell et al., 2008), and in clinical studies was the preferred choice of treatment for many patients (Wiegell et al., 2008). In addition to the reduced pain, patients were found to have equally efficient treatment outcomes compared to conventional PDT (Wiegell et al., 2008, 2012, 2013b, 2011b,a, 2013a, 2009). Another benefit of daylight PDT is that it is not limited by the size of the lesion, allowing larger areas to be treated simultaneously, thus reducing the required number of treatments for many patients. However, to continue to optimise the treatment parameters it is important to develop an appropriate approach to modelling daylight PDT.

To our knowledge, there have not been any theoretical investigations of daylight PDT and in this paper we present results from a 3D Monte Carlo Radiation Transfer (MCRT) model which simulates daylight PDT and investigates its potential and limitations. MCRT is a fast and accurate method for simulating the propagation of photons through a highly scattering medium and has, over the last two decades, become a popular technique to study the passage of light through skin tissue for a wide range of biomedical applications (Binzoni et al., 2008; Zhu and Liu, 2013; Swartling et al., 2003; Prahl et al., 1989). The code used to generate the presented results was adapted from a publically available code and was written in FORTRAN 77 (Wood, 2013) . The methods used in this work allow us to determine the photo-toxicity at different depths in the simulated skin and tumour tissue for different light sources and, in the case of daylight PDT, for different weather conditions.

The model adopts the same conditions for daylight PDT as for conventional PDT, where the PpIX is already accumulated before the tissue is exposed to light. The purpose of this simulation is to investigate daylight as a potential light source for PDT treatment keeping all other parameters the same as for conventional PDT.

\section{Methods}

MCRT is a numerical technique that uses the probabilistic nature of photon interactions to simulate the transport, scattering and absorption of light in highly scattering media. Photons from natural or artificial light sources enter skin and travel a distance to an interaction location that is determined by the scattering and absorption coefficients of that tissue type. The probability of photons being scattered is determined by the ratio $\mu_{\mathrm{s}} /\left(\mu_{\mathrm{a}}+\mu_{\mathrm{s}}\right)$, where $\mu_{\mathrm{s}}$ is the scattering coefficient and $\mu_{\mathrm{a}}$ is the absorption coefficient (Prahl et al., 1989; Swartling et al., 2003; Premru et al., 2013). The MCRT code that 
has been used in this work was originally developed for astronomy research (Wood and R. J. Reynolds, 1999) but was adapted and validated for skin tissue phantoms and PDT treatment simulations (Valentine et al., 2013, 2011).

Although the initial setup adopts tissue parameters that are uniform throughout the modelled region, MCRT was used (instead of analytic radiation transfer techniques) since photobleaching results in a non-uniform distribution of PpIX during the time dependent simulations.

\subsection{Optical Properties}

The MCRT code developed here can incorporate optical properties for different types of tissue, determined from theory or experiment. For the simulations presented in this paper, the wavelength dependent scattering $\left(\mu_{s}\right)$ and absorption coefficients $\left(\mu_{a}\right)$ for skin tissue and tumour tissue shown in figure 1 were adopted (Salomatina et al., 2006; Yudovsky and Pilon, 2010, 2011). Many studies report a wide range of values for the optical properties of human skin and are summarised in recent review papers by Lister et al (Lister et al., 2012) and Jacques (Jacques, 2013).

In this work, the model developed by Yudovsky et al (Yudovsky and Pilon, 2010, 2011) was employed. This model is based on in vivo measurements, and determines the optical properties for healthy skin tissue over the wavelength range required for daylight PDT modelling. The scattering coefficients for the tumour tissue (nodular basal cell carcinoma, nBCC) were taken from the model developed by Jacques which is based on the data from Salomatina et al (Jacques, 2013; Salomatina et al., 2006). The absorption coefficients for the tumour tissue are based on data determined by in vitro measurements of nBCC (Salomatina et al., 2006).

The angular scattering phase function was modelled using the Heyney-Greenstein phase function (Prahl et al., 1989; Henyey and Greenstein, 1941; Jacques et al., 1987; Van Gemert et al., 1989),

$$
H G(\theta)=\frac{1}{4 \pi} \frac{1-g^{2}}{\left(1+g^{2}-2 g \cos \theta\right)^{3 / 2}}
$$

where $\theta$ is the scattering angle and $g$ is the anisotropy factor which is in the range $-1 \leq g \leq 1$, with $g=0$ for isotropic scattering. For skin tissue, the scattering processes are very forward directed and we adopted a wavelength dependent anisotropy factor (Van Gemert et al., 1989), $g=0.62+0.29 \times 10^{-3} \lambda$, where $\lambda$ is the wavelength in nm. Polarisation is not considered and the reflectance at the air/tissue interface was determined using Fresnel reflectance assuming a uniform refractive index of 1.38 for the skin surface.

The absorption properties of PpIX affect the photodynamic dose and thus the efficacy of different PDT treatments. The wavelength dependent absorption of the photosensitive chemical PpIX is shown in figure 2 (Valentine et al., 2011, 2013; Valentine, 2011). 


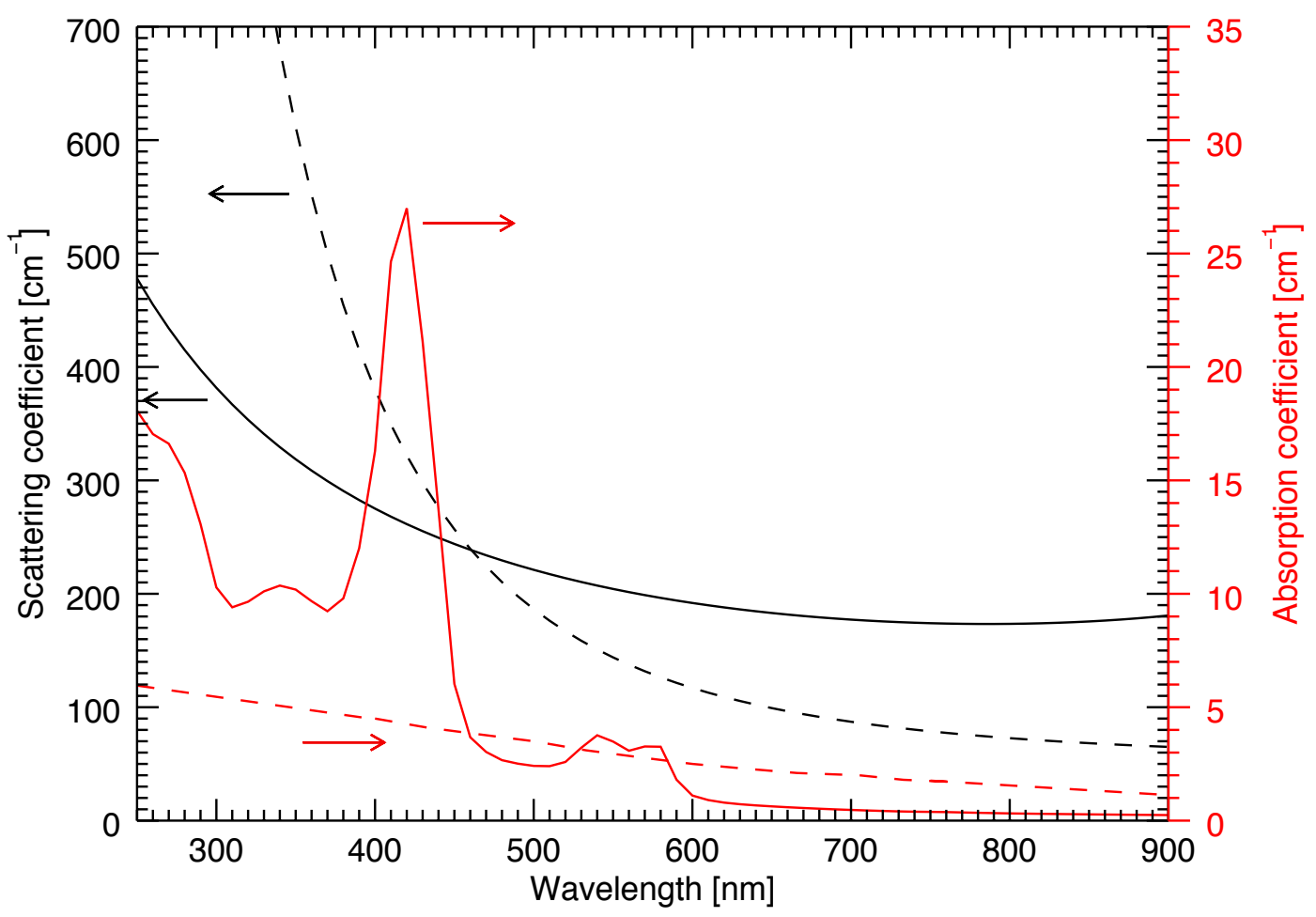

Figure 1. Wavelength dependent optical properties of tumour and skin tissue. The black curves correspond to the scattering coefficients (solid line corresponds to healthy skin tissue and dashed line corresponds to nodular BCC tissue). The red curves, plotted on the right axis, indicate the absorption coefficients for both healthy and nodular BCC tissue. The optical properties for healthy skin tissue are based on a modelling approach developed by Yudovsky et al (Yudovsky and Pilon, 2010, 2011) which takes into account different contributions of different chromophores. The scattering coefficients for tumour tissue are based on the interpolation of data extracted from Salomatina et al (Salomatina et al., 2006) using the approach developed by Jacques (Jacques, 2013)

\subsection{Some Code Details}

The MCRT code tracks power packets (hereafter referred to as "photons") on their random walk through a 3D linear cartesian grid. The source power is split among $\mathrm{N}$ Monte Carlo packets so that each packet has a radiant power given by $\mathrm{P} / \mathrm{N}$, where $\mathrm{P}$ is the source power (W). The optical properties can be changed for each individual grid cell, allowing for light propagation to be studied in complex 3D structures. In this study a cylindrical tumour surrounded by healthy tissue was investigated. The tumour was assumed to have a diameter of $6 \mathrm{~mm}$ and was positioned in the middle of a cartesian grid with dimension $10 \mathrm{~mm} \times 10 \mathrm{~mm}$ x 10mm. Repeated boundary conditions were adopted, 


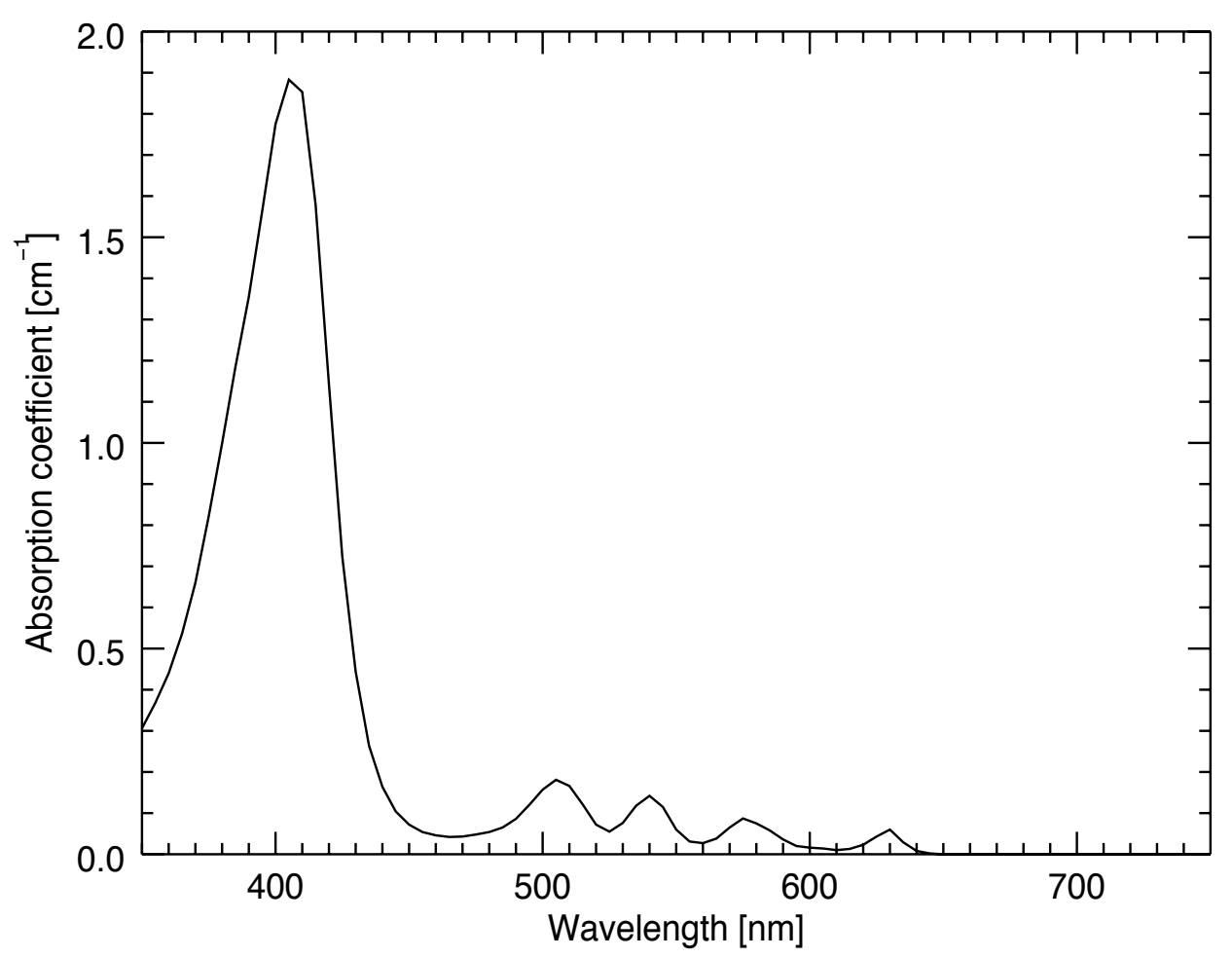

Figure 2. Absorption coefficient of PpIX. The absorption coefficient is concentration dependent and for this simulation we assume an initial number density of PpIX molecules of $1.62 \times 10^{14} \mathrm{~cm}^{-3}$. (Valentine, 2011; Valentine et al., 2013)

where photons that escape out of the sides of the grid continued in the same trajectory from the opposite side. The tissue outside of the tumour was assumed to be of a single tissue type (dermis) with a smooth uniform structure with different optical properties compared to the tumour. The PpIX was also assumed to have a uniform distribution within the cylindrical tumour.

Source photons were launched from the top of the grid and assigned an initial direction and wavelength depending on the source being simulated (e.g. daylight or an artificial light source). They were followed until they were either absorbed or scattered out of the simulation grid (figure 3). Absorbed photons could be re-emitted at a different wavelength as fluorescent emission (Swartling et al., 2003; Valentine et al., 2011; Farrell et al., 1998). A time dynamic was added to the code to take into account the photobleaching that changes the spatial distribution of PpIX throughout the treatment. At each time step $5 \times 10^{6}$ photons were launched and the photobleaching was determined before the simulated time was increased and the process was repeated. 
Photobleaching depends on the fluence rate as well as the initial PpIX concentration (Jacques et al., 1993; Farrell et al., 1998; Robinson et al., 1998; Valentine et al., 2011; Jongen and Sterenborg, 1997) and is described by,

$$
C(x, y, z, t)=C_{0}(x, y, z) e^{-\Psi(x, y, z) t / \beta(\lambda)}
$$

where $C(x, y, z, t)$ is the time dependent PpIX concentration at location $(x, y, z)$ at time $t, C_{0}(x, y, z)$ is the initial concentration, and $\Psi(x, y, z)$ is the local fluence rate in $\mathrm{Wcm}^{-2}$. The fluence rate was updated at each time step to take into account the change in PpIX concentration due to photobleaching. The motivation for generating a time dependent MCRT model is that the concentration of PpIX, which changes with time, affects the photodynamic dose (PDD).

The photobleaching constant, $\beta$, is defined as the light dose $\left(\mathrm{Jcm}^{-2}\right)$ required to reduce the fluorescence signal from the photosensitiser by $37 \%(1 / e)$. The value for $\beta$ has previously been established to be $14 \mathrm{Jcm}^{-2}$ for $630 \mathrm{~nm}$ irradiation (Valentine et al., 2011). However, research by other groups indicates that the photobleaching rate is wavelength dependent (Jacques et al., 1993; Nadeau et al., 2004). Hence, for daylight PDT, a wavelength dependent photobleaching constant has to be adopted. To our knowledge there is no information about how the photobleaching constant changes with wavelength. The assumption that has been made is that $\beta$ is inversely proportional to the absorption of the photosensitizer. This allows the photobleaching equation to include the difference in absorption for different wavelengths and to account for the different energies carried by photons of different wavelengths. Since we only have clinical data verifying the photobleaching constant at $630 \mathrm{~nm}$, for the simulations presented here the following wavelength dependence was applied to the photobleaching constant

$$
\beta(\lambda)=\frac{\beta_{630} \mu_{a 630} 630}{\lambda \mu_{a \lambda}}
$$

where $\beta_{630}=14 \mathrm{~J} \mathrm{~cm}^{-2}$ (clinically determined (Valentine et al., 2011)), $\mu_{\mathrm{a} 630}$ is the absorption coefficient of PpIX at $630 \mathrm{~nm}, \lambda$ is the wavelength of the simulated photon in $\mathrm{nm}$ and $\mu_{\mathrm{a} \lambda}$ is the absorption coefficient for PpIX at that wavelength. The photobleaching equation (Valentine et al., 2011; Jacques et al., 1993) assumes an exponential decay and an unlimited oxygen supply.

The PDD was calculated in the model and is defined as the number of photons absorbed by the photosensitiser, per cubic centimetre (Jacques et al., 1993). Following the method by Farrell et al (Farrell et al., 1998) the PDD was assumed to be proportional to the production of singlet oxygen. A toxic threshold determines the limit of the number of photons that have to be absorbed for necrosis to occur and has previously (Patterson et al., 1990) been found to be $8.6 \times 10^{17}$ photons $\mathrm{cm}^{-3}$. Although this threshold is based on measurements of Photofrin in liver tissue it was adopted for illustrative purposes, allowing for comparison with previously published models (Jacques et al., 1993; Valentine et al., 2011).

In the Monte Carlo model developed here, the wavelengths of the incident photons were chosen such that the probability distribution function of the irradiance, and 
therefore the daylight and Aktilite spectra, are reproduced. As the photons are tracked on their random walks within the model, they contribute to counters that contain the total fluence rate and PDD, which are output after each time step. The code was thoroughly verified and a more detailed description of the code and its validation can be found elsewhere (Valentine et al., 2013, 2011; Wood and R. J. Reynolds, 1999).

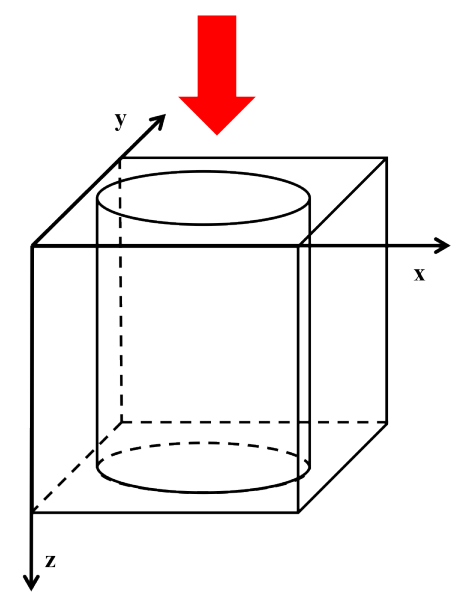

Figure 3. The MCRT simulation employs a 3D cartesian grid (100 grid cells on a side) which is illuminated from above. The optical properties are determined in each individual grid cell. A cylindrical tumour is added in the centre of the grid, where the PpIX is accumulated and assumed to be of uniform density. The dimensions of the grid are $10 \mathrm{~mm}$ on a side and the tumour diameter is $6 \mathrm{~mm}$.

\subsection{Natural and Artificial Light Sources}

The light distribution under the surface of the skin depends on the incident light field which in the case of daylight PDT consists of both direct and diffuse sunlight (figure 4). Due to the differences of the spectral and angular illumination between the diffuse and the direct light, it is important to decompose their relative contributions (Usami and Kawasaki, 2012). The light distribution also depends on weather conditions, time of the day and month of the year (Bird and Riordan, 1986). In the simulations it was assumed that the direct sunlight enters the skin at a constant angle depending on the latitude on Earth. The diffuse radiation, which we assumed illuminates the skin isotropically, comprises sunlight that has been scattered by molecules and particles in the atmosphere and reaches the surface of the Earth. The intensity of daylight is often measured in lux and is referred to as illuminance which is a measure of the irradiance weighted by the luminosity function, which takes into account the sensitivity of the human eye. The illuminance for daylight (at midday) varies from about 120000 lux for a very clear sunny day to around 20000 lux for an overcast day during summer, to considerably lower ( $<7500$ lux) in the middle of winter (overcast) (Wiegell et al., 2012). In this study we considered weather conditions in the middle of summer where the solar zenith angle 


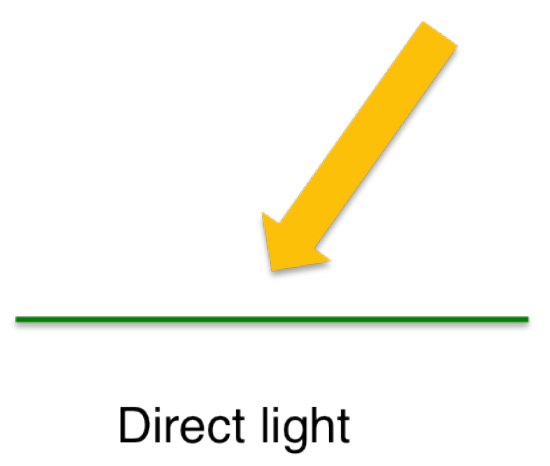

defined direction

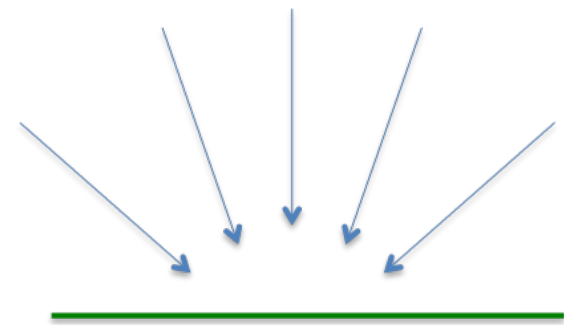

Diffuse Light

isotropic illumination

Figure 4. Daylight consists of both direct and diffuse light. Direct photons in the MCRT simulation are assigned a single angle of incidence. Diffuse light isotropically illuminates the surface of the skin phantom and consists of photons that have been scattered from particles and aerosols in the atmosphere. On sunny days the proportion of direct light will be larger and on an overcast day $100 \%$ of the daylight is assumed to be diffuse light.

was assumed to be $30^{\circ}$ at 56 degrees North (Van Heuklon, 1979), appropriate for the latitudes of Copenhagen in Denmark and Dundee in Scotland.

The direct and diffuse spectra used in the simulations were generated from a model of the solar spectrum reaching Earth (Bird and Riordan, 1986). The total spectral irradiance $\mathrm{I}_{\mathrm{T} \lambda}$ at wavelength $\lambda$, incident on a horizontal surface is given by,

$$
I_{T \lambda}=I_{d \lambda} \cos \theta+I_{s \lambda}
$$

where $I_{d \lambda}$ and $I_{s \lambda}$ are the direct and diffuse (scattered) contributions, and $\theta$ is the angle from the normal to the surface to the incident light. The direct component is dependent on the solar zenith angle and is expressed by,

$$
I_{d \lambda}=H_{o \lambda} D T_{r \lambda} T_{a \lambda} T_{w \lambda} T_{o \lambda} T_{u \lambda}
$$

where $H_{o \lambda}$ is the extraterrestrial irradiance at the mean sun-earth distance. $D$ is the correction parameter that takes into account the distance between the sun and the earth which depends on the day of the year. The remaining components correspond to transmittance function of the atmosphere at different wavelengths. $T_{r \lambda}$ takes into account the molecular or Rayleigh scattering, $T_{a \lambda}$ the aerosol attenuation, $T_{w \lambda}$ the water vapour absorption, $T_{o \lambda}$ the ozone absorption and $T_{u \lambda}$ takes into account the uniform mixed gas absorption.

The contribution from diffuse sunlight is

$$
I_{s \lambda}=I_{r \lambda}+I_{a \lambda}+I_{g \lambda}
$$

where $I_{r \lambda}$ is the Rayleigh scattering component, $I_{a \lambda}$ is the contribution from aerosol scattering, and the third component, $I_{g \lambda}$, takes into account the reflection of sunlight between the ground and the air. A more detailed description of the algorithm and model 
can be found in Bird et al (1986) (Bird and Riordan, 1986). The exposure will vary between different people and different body sites (Diffey, 2008; Schauberger, 1990). To take this into account different surface orientations were investigated which will alter the intensity of the irradiance according to equation 4.

The direct and diffuse spectra that were input to the Monte Carlo simulations (figure 5) represent conditions for midday on a clear summer day. The spectra are generated with a resolution of $5 \mathrm{~nm}$. In this model the direct light represents $80 \%$ of the total illuminance of about 100000 lux. For daylight PDT simulations of an overcast day, the spectrum was assumed to be $100 \%$ diffuse light, corresponding to about 15 000 lux. Daylight PDT was compared to conventional PDT by simulating illumination by an Aktilite lamp (Photocure ASA, Hoffsveien 48, N-0377 Oslo, Norway) (Moseley, 2005) which is a red LED light source, typically used for conventional PDT treatment. The spectral irradiance for the Aktilite is plotted in figure 5. Also shown in figure 5 is the spectral shape of the PpIX absorption spectrum, showing the strong absorption around $400 \mathrm{~nm}$ and the smaller absorption peak coincident with the Aktilite spectrum.

\section{Results for Monte Carlo Modelling of daylight PDT}

Daylight may be absorbed by PpIX over a wide wavelength range, while the Aktilite targets the smaller PpIX absorption peak around $630 \mathrm{~nm}$ (figure 5). This means that the total absorbed energy (by PpIX) is roughly 5 times greater for daylight compared to the Aktilite. The conventional treatment light source targets the PpIX absorption peak at $630 \mathrm{~nm}$ because of the deeper penetration depths attainable by long wavelength light (Richards-Kortum and Sevick-Muraca, 1996). The dominant absorbing wavelengths for daylight and conventional PDT are $405 \mathrm{~nm}$ and $630 \mathrm{~nm}$ respectively and in figure 6 results of two separate Monte Carlo simulations for the PDD assuming normal illumination by monochromatic light at $405 \mathrm{~nm}$ and $630 \mathrm{~nm}$ are shown. Results are displayed for four different light doses of 10, 20, 40 and $75 \mathrm{Jcm}^{-2}$. As one would expect, due to the deeper penetration, the $630 \mathrm{~nm}$ light achieves higher PDD at greater depths compared to the $405 \mathrm{~nm}$ light. Although not penetrating to as great a depth, the 405 nm light achieves the same PDD at superficial layers.

The results of Monte Carlo simulations of the depth dependent PDD using the daylight spectrum (on clear and overcast days) and Aktilite are shown in figure 7 for light doses of 10 and $75 \mathrm{Jcm}^{-2}$, with a PpIX absorption coefficient at $630 \mathrm{~nm}$ obtained from figure 2 of $0.06 \mathrm{~cm}^{-1}$. To investigate the full range of published values (Farrell et al., 1998), a higher and lower PpIX concentration were also investigated. Figures 8 and 9 show the resulting PDD for daylight PDT when the PpIX absorption coefficients at $630 \mathrm{~nm}$ are $0.18 \mathrm{~cm}^{-1}$ and $0.02 \mathrm{~cm}^{-1}$, representing higher and lower concentrations of PpIX compared to the results in figure 7 .

Figure 6 - 8 show simulations for the case when direct daylight is incident at $\theta=30^{\circ}$ from the normal to the skin surface. The results of different surface orientations are shown in figure 10. For the clear daylight situation the PDD has been simulated for $\theta$ in 


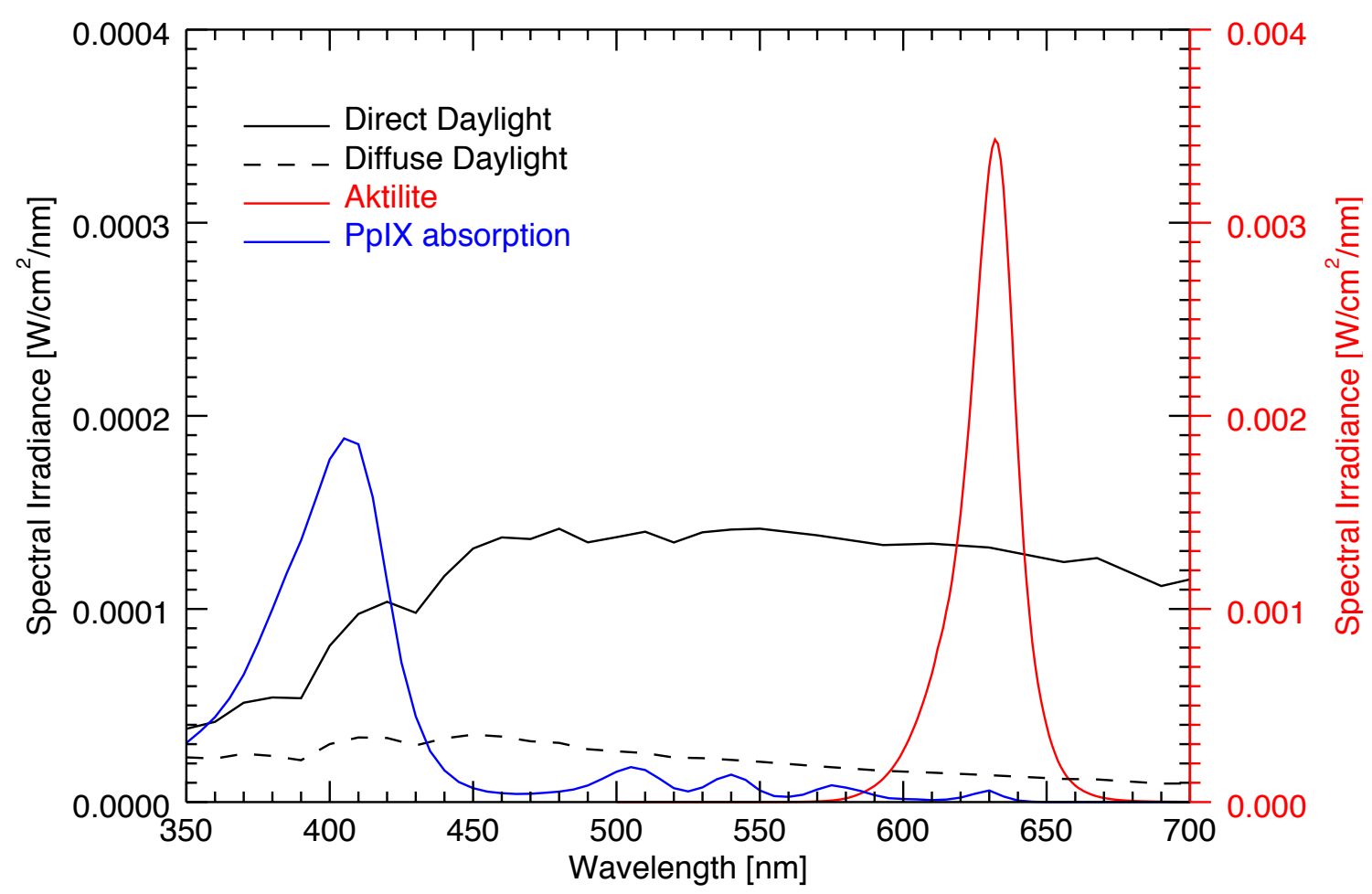

Figure 5. Illuminating spectra adopted for our MCRT simulations for daylight (Bird and Riordan, 1986) and the Aktilite (Moseley, 2005). The indicative absorption curve for PpIX is shown in blue and is provided as a guide to the eye only to highlight the dominant absorbing features. The Aktilite spectrum targets a small absorption peak around $630 \mathrm{~nm}$ while the daylight utilises the whole absorption spectrum of PpIX which results in more energy being absorbed from the daylight even though the spectral irradiance is lower.

equation 4 , of $0^{\circ}, 60^{\circ}$ and $80^{\circ}$. All the situations investigated assumed that the patient would orient the lesion being treated towards the sun.

\section{Discussion}

Due to the Soret band of PpIX (the broad absorption around 400nm in figure 2) and limited penetration depth at these wavelengths, the daylight will contribute to a larger accumulated PDD closer to the surface. This results in rapid photobleaching at the beginning of the treatment that then slows as treatment progresses since the concentration of PpIX is reduced. Figure 7 indicates that on a clear sunny day it would take about 30 minutes to deliver a light dose of $75 \mathrm{Jcm}^{-2}$ with an effective treatment 


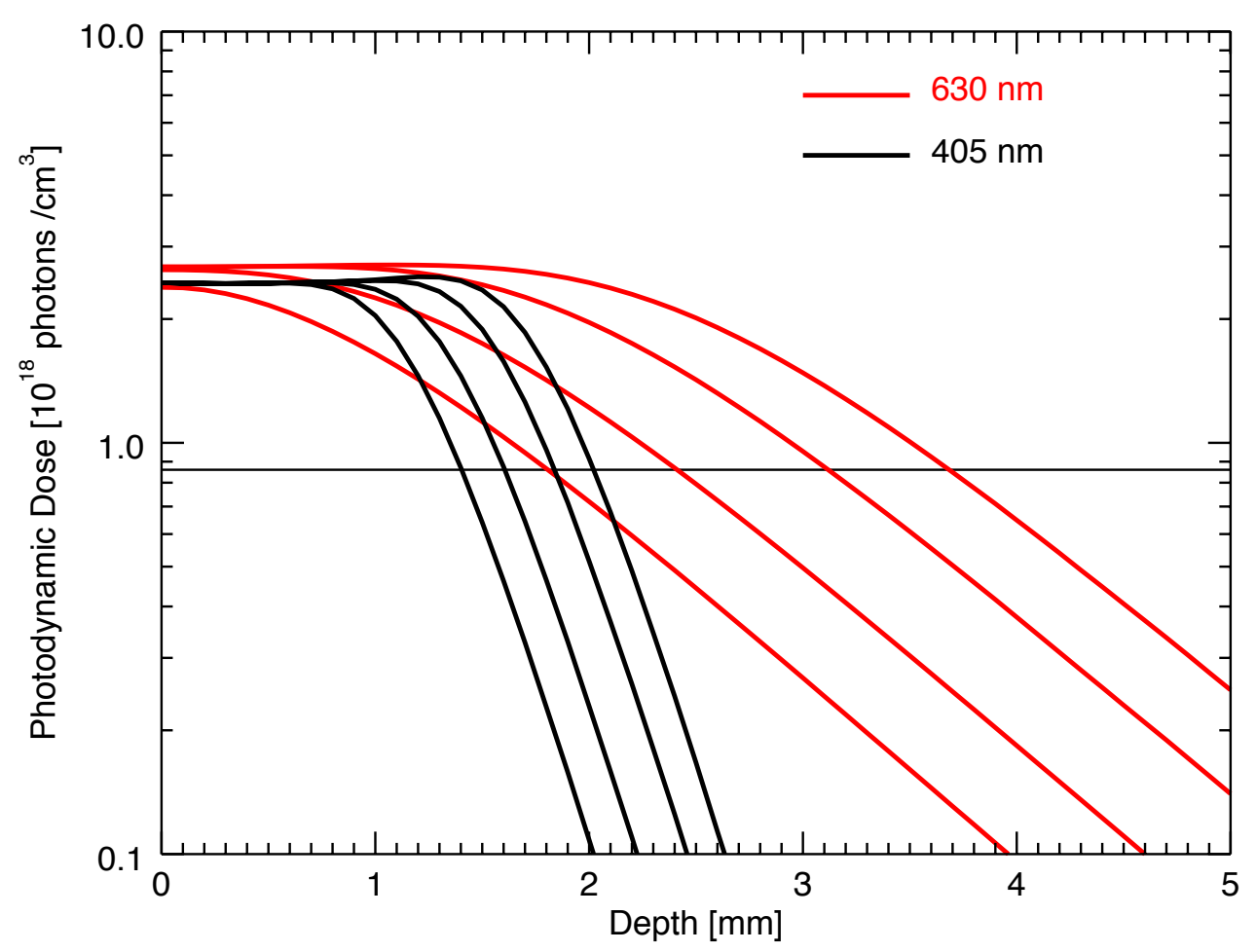

Figure 6. Photodynamic dose for two separate wavelengths at normal incidence on the tumour. Four different light doses are plotted, 10, 20, 40 and $75 \mathrm{Jcm}^{-2}$, where both light sources are assumed to have an irradiance of $82 \mathrm{mWcm}^{-2}$. The horizontal line corresponds to the toxic threshold which determines the number of photons that have to be absorbed for necrosis to occur.

depth of over $2 \mathrm{~mm}$. The same light dose would take about $2.5 \mathrm{~h}$ to deliver on an overcast day with approximately the same effective treatment depth. The Aktilite delivers the same light dose after about 15 min of irradiation, yielding an effective treatment depth of about $3 \mathrm{~mm}$. It is clear from figure 7 that the treatment depth is limited by the light penetration. Even though a longer treatment time results in deeper treatment, this requires sufficient amount of PpIX and light.

The skin phantom we adopt is a simplified model, where the well defined layered structure has not been taken into account. The shape of the tumour is not an exact replica of those found in patients but shows the discrepancy between healthy tissue and diseased tissue. The optical properties are assumed to be uniform within the tissue and future developments could include different structures such as blood vessels that may affect the spatial distribution of absorbers and scatterers (Finlay and Foster, 2004; Rajaram et al., 2010). 


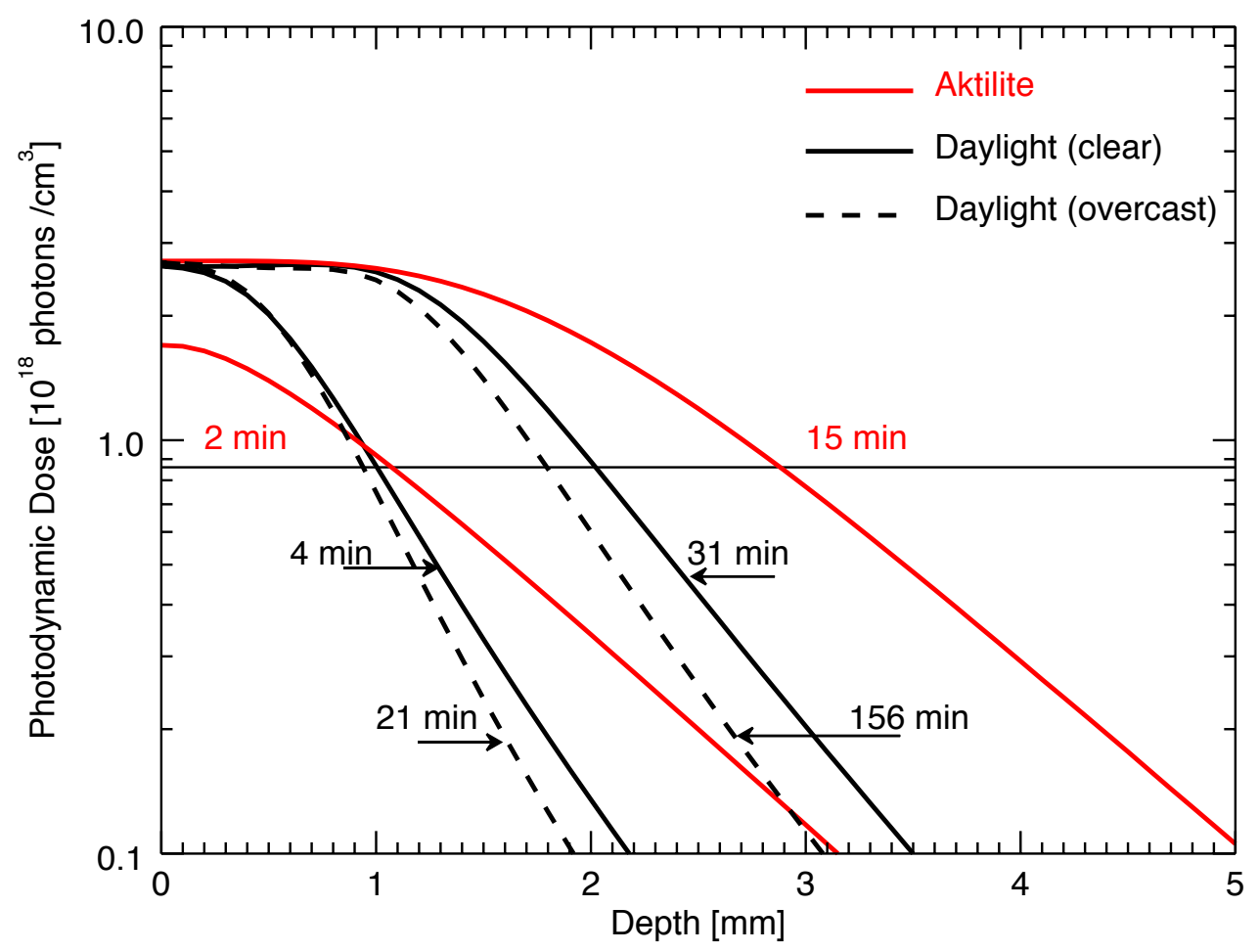

Figure 7. Photodynamic dose for conventional PDT treatment (red), and for daylight PDT for both a clear summer day (solid black) as well as an overcast summer day (dashed black). The photodynamic dose is plotted for two light doses, 10 and 75 $\mathrm{Jcm}^{-2}$. The irradiance for the Aktilite is assumed to be $82 \mathrm{mWcm}^{-2}$, while the irradiance for a clear summer day is determined to be $41 \mathrm{mWcm}^{-2}$ and for an overcast summer day the irradiance is determined to be $8 \mathrm{mWcm}^{-2}$. Corresponding treatment times are displayed in the plot.

A further complication in clinical treatment is the time dependent build up of PpIX prior to the commencement of irradiation. In this work a uniform distribution of PpIX was assumed, although treatment depth may also be limited by the distribution of PpIX (Svaasand et al., 1996; Star et al., 2002).

Even though studies have shown that oxygen is depleted during treatment, the model presented here assumes an unlimited oxygen supply. To accurately take into account the oxygen supply (Tyrrell et al., 2011; Liu et al., 2010; Henderson et al., 2000) both oxygen consumption as well as perfusion would have to be considered. This phenomenon is not well understood and further investigation is required (Tyrrell et al., 2011).

It is well known that optical properties vary for patients with different skin types 


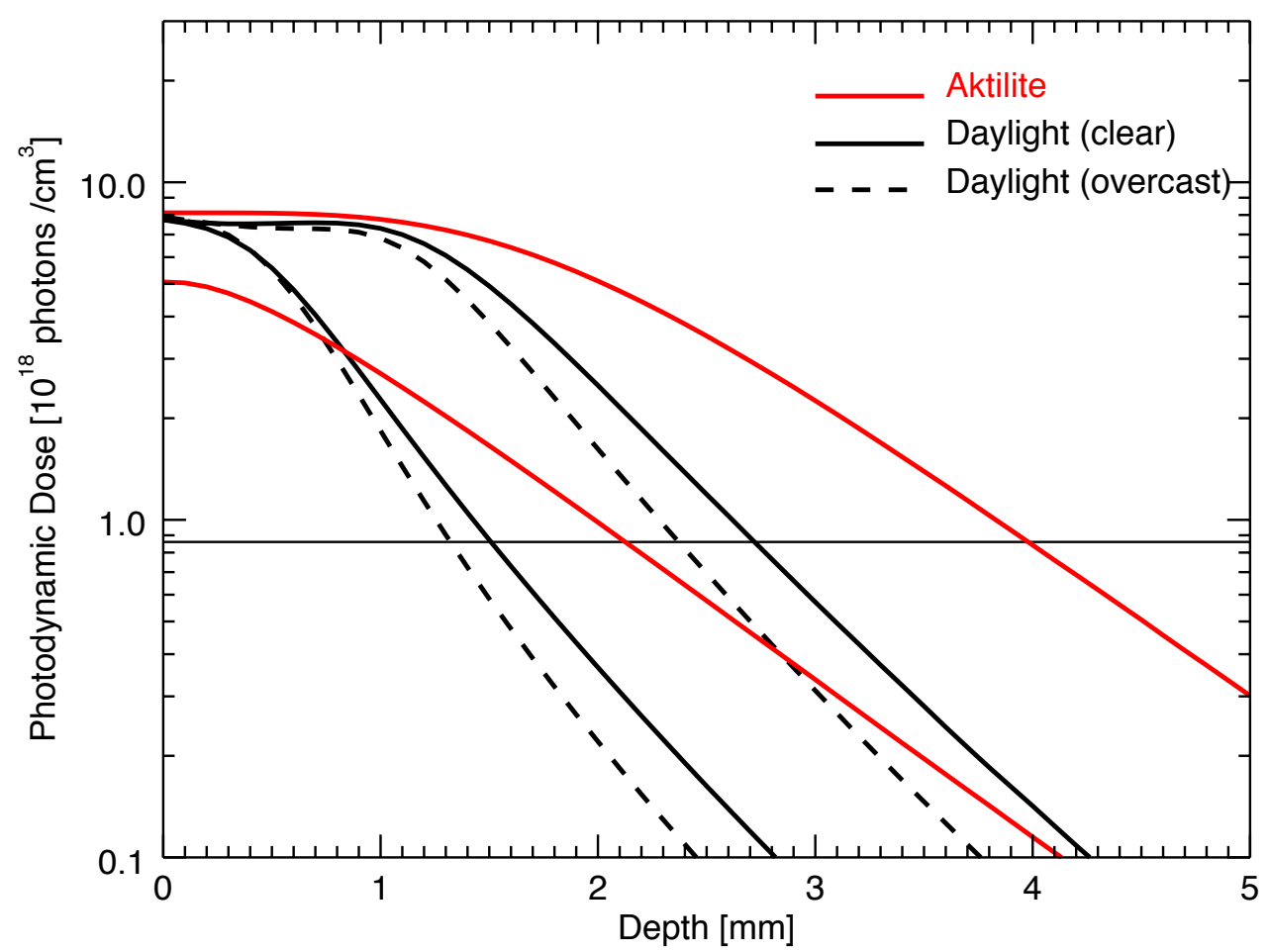

Figure 8. Photodynamic dose for the situation where the concentration of PpIX has been increased by a factor of three. This corresponds to an absorption coefficient at $630 \mathrm{~nm}$ for PpIX of $0.18 \mathrm{~cm}^{-1}$. Light doses of 10 and $75 \mathrm{Jcm}^{-2}$ are plotted and correspond to the same treatment times as indicated in figure 7 .

and may be a reason for the different treatment outcomes. In our simulations, apart from the time dependent effects of photobleaching on the concentration of PpIX, all other optical properties remain constant during treatment. Other effects such as heating and oxygen consumption during treatment may change the optical properties and affect the treatment depth.

It is clear from figure 8 and 9 that the concentration, and therefore the uptake and accumulation of PpIX in the tumour, will have a large effect on the treatment outcome. Fewer available PpIX molecules will restrict the photon absorption but will also result in slower photobleaching. The absorption of PpIX will lead to generation of toxic oxygen species, hence the initial PpIX concentration may affect the treatment outcome as well.

Fluorescence is a quantity that can be used to determine the relative concentration of PpIX. This has been studied by Valentine et al (Valentine et al., 2013; Valentine, 2011), showing that the initial fluorescent signal varies dramatically among patients, 


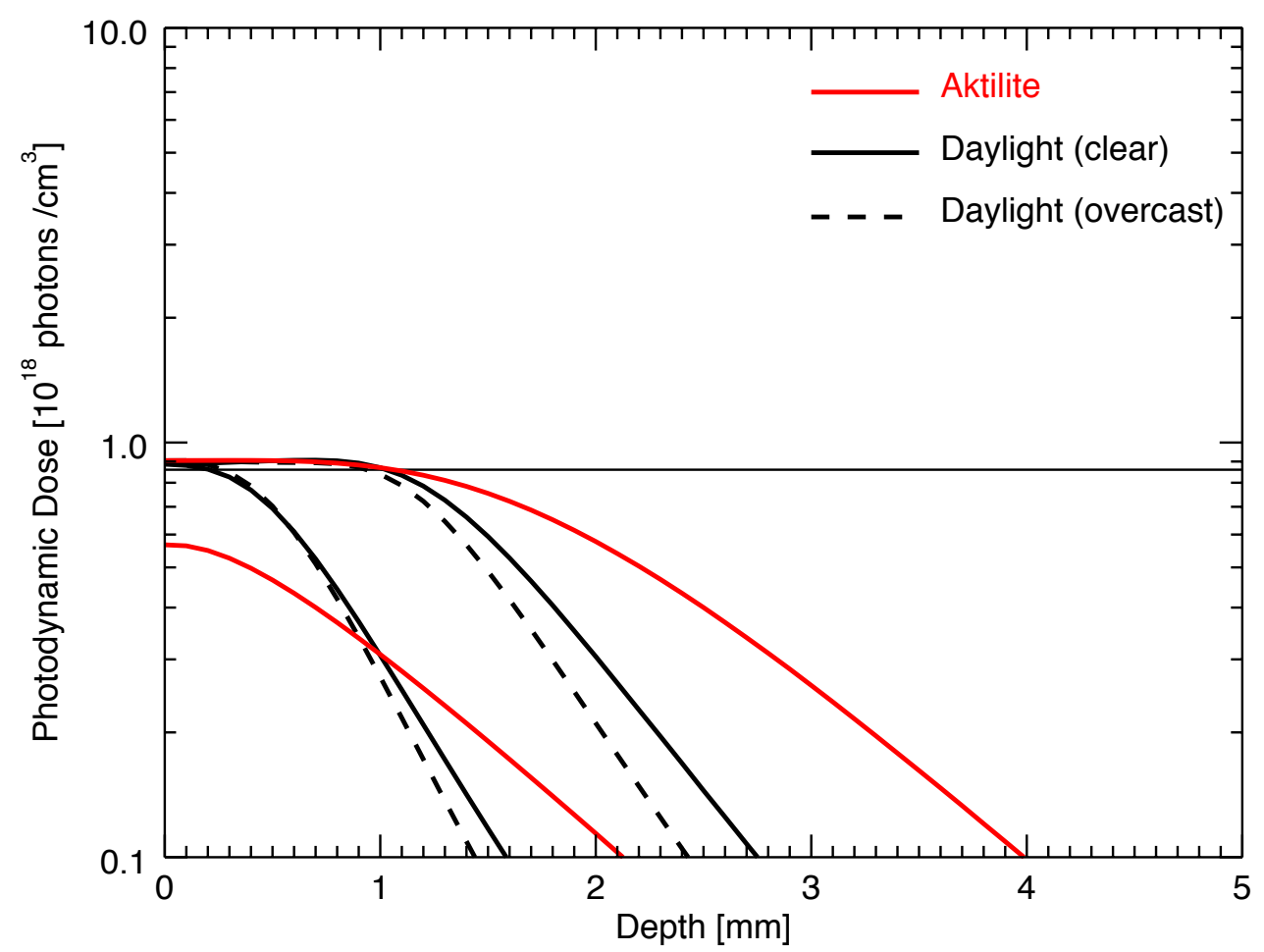

Figure 9. Photodynamic dose for the situation where the concentration of PpIX has been reduced by a factor of three. This corresponds to an absorption coefficient at $630 \mathrm{~nm}$ for PpIX of $0.02 \mathrm{~cm}^{-1}$. The light doses and treatment times are equivalent to those plotted in figure 7 and 8 .

suggesting that the uptake and accumulation of PpIX within the tumour will also be different from patient to patient.

A patient undergoing daylight PDT will not constantly orient the area of interest perpendicular to the direct sunlight since this will sometimes be inconvenient. Figure 10 shows that a steeper angle to the sun results in a lower irradiance and therefore a slightly lower effective penetration depth of the treatment light.

Although there are more complicated descriptions of photobleaching (Georgakoudi and Foster, 1998), the photobleaching equation that has previously been applied in the group was also adopted here (Valentine et al., 2013). However a modification to this equation was made where the PpIX absorption was included as well as a time-dependent fluence rate. For previously modelled photobleaching, where similar equations have been adopted, the photobleaching is only dependent on the local fluence rate as well as time. By including a wavelength dependent $\beta$, the photobleaching is not only dependent on 


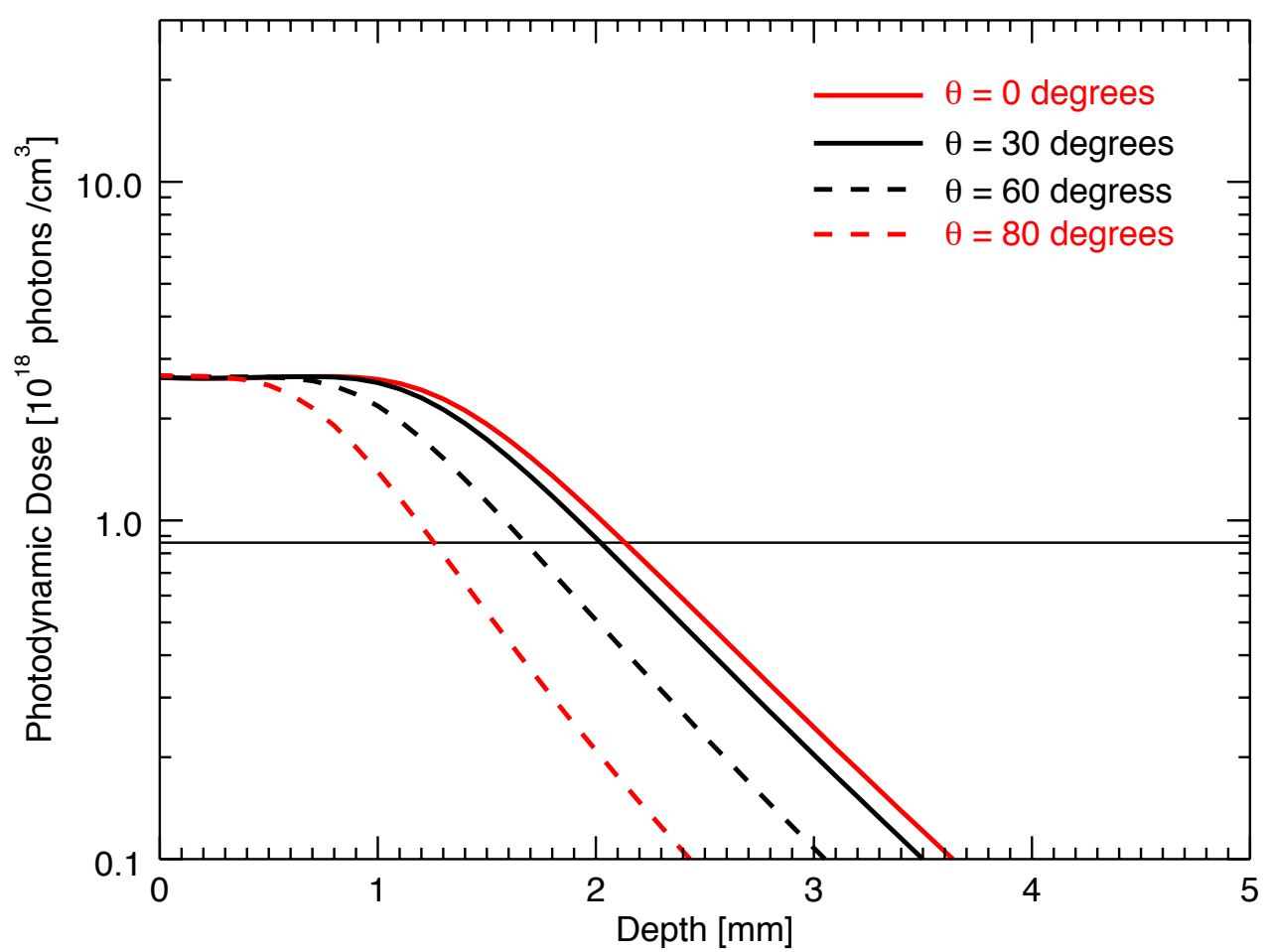

Figure 10. Effect of different illumination angles. The four different angles that have been investigated are $\theta=0^{\circ}, 30^{\circ}, 60^{\circ}$, and $80^{\circ}$, where $\theta$ is the angle between the incident light and the normal to the surface. In each case it is assumed that the person being exposed to daylight faces the sun.

time, but also depends on the absorption of PpIX. This ensures that when the PpIX is fully photobleached, the number of absorbed photons is the same, independent of irradiance and wavelength. This would not be the case for a wavelength independent value for $\beta$.

\section{Conclusions}

We have developed a MCRT model for the simulation of conventional and daylight activated PDT. It was used to investigate the potential and limitations of treating superficial skin lesions with artificial and natural light. The model determines the toxicity (PDD) within the tumour as a function of depth during different weather conditions. Adopting optical properties appropriate for healthy and diseased tissue, simulations show that for a clear summer day it would take around 30 minutes to deliver a light dose of $17 \mathrm{Jcm}^{-2}$, which is comparable to the light dose used in conventional 
PDT treatments. The simulations predict an effective treatment depth for daylight PDT of over $2 \mathrm{~mm}$, making it ideal for treating superficial skin lesions, though limiting its efficacy for deeper lying tumours. The effect of PpIX concentration was also investigated, finding that a higher concentration yields a greater effective penetration depth. The results of our MCRT simulations support the clinical findings that daylight PDT can be an effective alternative to conventional PDT for appropriate tumour types.

\section{Acknowledgments}

C L Campbell acknowledges financial support from an UK EPSRC PhD studentship 


\section{References}

Binzoni, T., Leung, T., Giust, R., Rufenacht, D., and Gandjbakhche, A. (2008). Light transport in tissue by $3 \mathrm{~d}$ monte carlo: Influence of boundary voxelization. Computer Methods and Programs in Biomedicine, 89(1):14 - 23.

Bird, R. and Riordan, C. (1986). Simple Solar Spectral Model for Direct and Diffuse Irradiance on Horizontal and Tilted Planes at the Earth's Surface for Cloudless Atmospheres. Journal of Applied Meteorology, 25:87-97.

Castano, A., Demidova, T., and Hamblin, M. (2004). Mechanisms in photodynamic therapy: part one - photosensitizers, photochemistry and cellular localization. Photodiagnosis and Photodynamic Therapy, 1(4):279 - 293.

Cottrell, W. J., Paquette, A. D., Keymel, K. R., Foster, T. H., and Oseroff, A. R. (2008). Irradiance-dependent photobleaching and pain in ?-aminolevulinic acid-photodynamic therapy of superficial basal cell carcinomas. Clinical Cancer Research, 14(14):44754483.

Darlenski, R. and Fluhr, J. (2012). Photodynamic therapy in dermatology: past, present, and future. Journal of Biomedical Optics, 18(6):061208-061208.

Diffey, B. (2008). A behavioral model for estimating population exposure to solar ultraviolet radiation. Photochemistry and Photobiology, 84(2):371-375.

Farrell, T., Hawkes, R., Patterson, M., and Wilson, B. (1998). Modeling of photosensitizer fluorescence emission and photobleaching for photodynamic therapy dosimetry. Appl. Opt., 37(31):7168-7183.

Finlay, J. and Foster, T. (2004). Effect of pigment packaging on diffuse reflectance spectroscopy ofsamples containing red blood cells. Opt. Lett., 29(9):965-967.

Georgakoudi, I. and Foster, T. (1998). Singlet oxygen-versus nonsinglet oxygen-mediated mechanisms of sensitizer photobleaching and their effects on photodynamic dosimetry. Photochemistry and Photobiology, 67(6):612-625.

Henderson, B., Busch, T., Vaughan, L., Frawley, N., Babich, D., Sosa, T., Zollo, J., Dee, A., Cooper, M., Bellnier, D., Greco, W., and Oseroff, A. (2000). Photofrin photodynamic therapy can significantly deplete or preserve oxygenation in human basal cell carcinomas during treatment, depending on fluence rate. Cancer Research, 60(3):525-529.

Henderson, B. and Dougherty, T. (1992). How does photodynamic therapy work? Photochemistry and Photobiology, 55(1):145-157.

Henyey, L. and Greenstein, J. (1941). Diffuse radiation in the Galaxy. , 93:70-83.

Jacques, S. (2013). Optical properties of biological tissues: a review. Physics in Medicine and Biology, 58(11):R37.

Jacques, S., Alter, C., and Prahl, S. (1987). Angular dependence of HeNe laser light scattering by human dermis. Lasers Life Sci., 1:309-333. 
Jacques, S., Joseph, R., and Gofstein, G. (1993). How photobleaching affects dosimetry and fluorescence monitoring of pdt in turbid media. Proc. SPIE, 1881:168-179.

Jongen, A. and Sterenborg, H. (1997). Mathematical description of photobleaching in vivo describing the influence of tissue optics on measured fluorescence signals. Physics in Medicine and Biology, 42(9):1701.

Lister, T., Wright, P., and Chappell, P. (2012). Optical properties of human skin. Journal of Biomedical Optics, 17(9):090901-1-090901-15.

Liu, B., Farrell, T. J., and Patterson, M. S. (2010). A dynamic model for ala-pdt of skin: simulation of temporal and spatial distributions of ground-state oxygen, photosensitizer and singlet oxygen. Physics in Medicine and Biology, 55(19):5913.

Moseley, H. (2005). Light distribution and calibration of commercial pdt led arrays. Photochem. Photobiol. Sci., 4:911-914.

Nadeau, V., O’Dwyer, M., Hamdan, K., Tait, I., and Padgett, M. (2004). In vivo measurement of 5-aminolaevulinic acid-induced protoporphyrin ix photobleaching: a comparison of red and blue light of various intensities. Photodermatology, Photoimmunology Photomedicine, 20(4):170-174.

Patterson, M. S., Wilson, B., and Graff, R. (1990). In vivo tests of the concept of photodynamic threshold dose in normal rat liver photosensitized by aluminum chlorosulphonated phthalocyanine. Photochemistry and Photobiology, 51(3):343-349.

Prahl, S., Keijzer, M., Jacques, S., and Welch, A. (1989). A monte carlo model of light propagation in tissue. In SPIE Proceedings of Dosimetry of Laser Radiation in Medicine and Biology, pages 102-111. Press.

Premru, J., Milani, M., and Majaron, B. (2013). Monte carlo simulation of radiation transfer in human skin with geometrically correct treatment of boundaries between different tissues. Proc. SPIE, 8579:85790Z-85790Z-13.

Rajaram, N., Gopal, A., Zhang, X., and Tunnell, J. (2010). Experimental validation of the effects of microvasculature pigment packaging on in vivo diffuse reflectance spectroscopy. Lasers in Surgery and Medicine, 42(7):680-688.

Richards-Kortum, R. and Sevick-Muraca, E. (1996). Quantitative optical spectroscopy for tissue diagnosis. Annual Review of Physical Chemistry, 47(1):555-606. PMID: 8930102.

Robinson, D., de Bruijn, H., van der Veen, N., Stringer, M., Brown, S., and Star, W. (1998). Fluorescence photobleaching of ala-induced protoporphyrin ix during photodynamic therapy of normal hairless mouse skin: the effect of light dose and irradiance and the resulting biological effect. Photochem Photobiol, 67(1):140-9.

Salomatina, E., Jiang, B., Novak, J., and Yaroslavsky, A. N. (2006). Optical properties of normal and cancerous human skin in the visible and near-infrared spectral range. Journal of Biomedical Optics, 11(6):064026-064026-9. 
Schauberger, G. (1990). Model for the global irradiance of the solar biologicallyeffective ultraviolet-radiation on inclined surfaces. Photochemistry and Photobiology, 52(5):1029-1032.

Star, W. M., Aalders, M. C. G., Sac, A., and Sterenborg, H. J. C. M. (2002). Quantitative model calculation of the time-dependent protoporphyrin ix concentration in normal human epidermis after delivery of ala by passive topical application or iontophoresis. Photochemistry and Photobiology, 75(4):424-432.

Svaasand, L. O., Wyss, P., Wyss, M.-T., Tadir, Y., Tromberg, B. J., and Berns, M. W. (1996). Dosimetry model for photodynamic therapy with topically administered photosensitizers. Lasers in Surgery and Medicine, 18(2):139-149.

Swartling, J., Pifferi, A., Enejder, A., and Andersson-Engels, S. (2003). Accelerated monte carlo models to simulate fluorescence spectra from layered tissues. J. Opt. Soc. Am. A, 20(4):714-727.

Tyrrell, J., Thorn, C., Shore, A., Campbell, S., and Curnow, A. (2011). Oxygen saturation and perfusion changes during dermatological methylaminolaevulinate photodynamic therapy. British Journal of Dermatology, 165(6):1323-1331.

Usami, A. and Kawasaki, N. (2012). Modeling of solar spectral irradiance data from cloudless to overcast skies. Japanese Journal of Applied Physics, 51:10NF06.

Valentine, R. (2011). Biological aspects of photodynamic therapy. Ph.D. Thesis, University of St Andrews.

Valentine, R., BRown, C., Moseley, H., Ibbotson, S., and Wood, K. (2011). Monte carlo modeling of in vivo protoporphyrin ix fluorescence and singlet oxygen production during photodynamic therapy for patients presenting with superficial basal cell carcinomas. Journal of Biomedical Optics, 16(4):048002-048002-11.

Valentine, R. M., Ibbotson, S., Wood, K., Brown, C., and Moseley, H. (2013). Modelling fluorescence in clinical photodynamic therapy. Photochem. Photobiol. Sci., 12:203213.

Van Gemert, M., Jacques, S., Sterenborg, H., and Star, W. M. (1989). Skin optics. Biomedical Engineering, IEEE Transactions on, 36(12):1146-1154.

Van Heuklon, T. (1979). Estimating atmospheric ozone for solar radiation models. Solar Energy, 22(1):63 - 68.

Wachowska, M., Muchowicz, A., Firczuk, M., Gabrysiak, M., Winiarska, M., Wanczyk, M., Bojarczuk, K., and Golab, J. (2011). Aminolevulinic acid (ala) as a prodrug in photodynamic therapy of cancer. Molecules, 16(5):4140-4164.

Wiegell, S., Fabricius, S., Heydenreich, J., Enk, C., Rosso, S., Baumler, W., Baldursson, B., and Wulf, H. (2013a). Weather conditions and daylight-mediated photodynamic therapy: protoporphyrin ix-weighted daylight doses measured in six geographical locations. British Journal of Dermatology, 168(1):186-191.

Wiegell, S., Fabricius, S., Stender, I., Berne, B., Kroon, S., Andersen, B., Mork, C., Sandberg, C., Jemec, G., Mogensen, M., Brocks, K., Philipsen, P., Heydenreich, J., 
Hdersdal, M., and Wulf, H. (2011a). A randomized, multicentre study of directed daylight exposure times of $11 / 2$ vs. $21 / 2 \mathrm{~h}$ in daylight-mediated photodynamic therapy with methyl aminolaevulinate in patients with multiple thin actinic keratoses of the face and scalp. British Journal of Dermatology, 164(5):1083-1090.

Wiegell, S., Hadersdal, M., Eriksen, P., and Wulf, H. (2009). Photodynamic therapy of actinic keratoses with $8 \%$ and $16 \%$ methyl aminolaevulinate and home-based daylight exposure: a double-blinded randomized clinical trial. British Journal of Dermatology, 160(6):1308-1314.

Wiegell, S., Hadersdal, M., Philipsen, P., Eriksen, P., Enk, C., and Wulf, H. (2008). Continuous activation of ppix by daylight is as effective as and less painful than conventional photodynamic therapy for actinic keratoses; a randomized, controlled, single-blinded study. British Journal of Dermatology, 158(4):740-746.

Wiegell, S., Heydenreich, J., Fabricius, S., and Wulf, H. (2011b). Continuous ultra-lowintensity artificial daylight is not as effective as red led light in photodynamic therapy of multiple actinic keratoses. Photodermatology, Photoimmunology Photomedicine, 27(6):280-285.

Wiegell, S., Skodt, V., and Wulf, H. (2013b). Daylight-mediated photodynamic therapy of basal cell carcinomas - an explorative study. Journal of the European Academy of Dermatology and Venereology.

Wiegell, S., Wulf, H., Szeimies, R.-M., Basset-Seguin, N., Bissonnette, R., Gerritsen, M.-J., Gilaberte, Y., Calzavara-Pinton, P., Morton, C., Sidoroff, A., and Braathen, L. (2012). Daylight photodynamic therapy for actinic keratosis: an international consensus. Journal of the European Academy of Dermatology and Venereology, 26(6):673-679.

Wilson, B. and Patterson, M. (2008). The physics, biophysics and technology of photodynamic therapy. Physics in Medicine and Biology, 53(9):R61.

Wood, K. (2013). http://www-star.st-and.ac.uk/ kw25/. Monte Carlo Radiation Transfer.

Wood, K. and R. J. Reynolds, R. (1999). A model for the scattered light contribution and polarization of the diffuse $\mathrm{h}$ galactic background. The Astrophysical Journal, $525(2): 799$.

Yudovsky, D. and Pilon, L. (2010). Rapid and accurate estimation of blood saturation, melanin content, and epidermis thickness from spectral diffuse reflectance. Appl. Opt., 49(10):1707-1719.

Yudovsky, D. and Pilon, L. (2011). Retrieving skin properties from in vivo spectral reflectance measurements. Journal of Biophotonics, 4(5):305-314.

Zhu, C. and Liu, Q. (2013). Review of monte carlo modeling of light transport in tissues. Journal of Biomedical Optics, 18(5):050902-050902. 